\title{
The value of acoustic radiation force impulse elastography in differentiating cirrhotic and non-cirrhotic ascites
}

Objectives: Cirrhosis accounts for $85 \%$ of the causes of ascites besides other causes such as malignancy, heart failure, tuberculosis, etc. Ascitic fluid analysis is one of the most important tests to diagnose causes of ascites. However, this is an invasive procedure. Acoustic Radiation Force Impulse (ARFI) is a new measurement of elastography which can diagnose cirrhosis in patients with ascites. This study evaluates the value of ARFI for initial diagnosis of ascites due to cirrhosis or not due to cirrhosis.

Materials and methods: Institutional review board approved this cross-sectional study. The study was carried out on 90 patients with ascites. All patients diagnosed with ascites at Nguyen Trai hospital and Clinic of Pham Ngoc Thach University of Medicine were enrolled from January 2013 to October 2018. All patients underwent ARFI and ascitic fluid analysis.

Results: The rate of cirrhotic and non-cirrhotic etiology of ascites were $84.4 \%$ and $15.6 \%$, respectively The mean liver stiffness measurements by means of ARFI were statistically significantly higher in patients with cirrhotic ascites than in those with non-cirrhotic ascites: $3.84 \mathrm{~m} / \mathrm{s} \pm 1.43 \mathrm{v} / \mathrm{s} 1.48 \mathrm{~m} / \mathrm{s}$ \pm 0.55 ( $p<0.001$ ). In our study, AUROC had a validity of $92.1 \%$ with $95 \% \mathrm{Cl}=0.963-0.982$ to diagnose of cirrhotic ascites. For an optimal cut-off value of $2.2 \mathrm{~m} / \mathrm{s}$ for predicting cirrhosis and ascites in the context of cirrhosis, ARFI had sensitivity of $98 \%$, specificity of $66.7 \%$, positive predictive value of $94.3 \%$, negative predictive value of $85.7 \%$ for predicting cirrhotic ascites.

Conclusion: ARFI elastography was feasible in all patients with ascites. It had a high performance for diagnosis of cirrhosis (AUROC >0.9) and an effective differentiation between the cirrhotic and non-cirrhotic cause of ascites.

\section{KEYWORDS: ascites - liver stiffness - liver cirrhosis - acoustic radiation force impulse - elastography}

\section{Introduction}

ARFI is a novel assessment method that stimulates tissue by using a short pulse of 0.3 sec in an ROI of $10 \times 5 \mathrm{~mm}$, selected at a fixed transmission frequency of $2.67 \mathrm{MHz}$ without compression. Pulses passing through the targeted tissue at the ROI cause displacement and they will simultaneously return to its original location. The tissue displacement is measured by a conventional ultrasound beam with the same probe used to generate the force. The shear wave velocity (SWV) is positively proportional to the tissue stiffness (the faster SWV is, the stiffer tissue is). SWV is united as meters per second $(\mathrm{m} / \mathrm{s})$ and displayed on the screen [1]. ARFI has many advantages over transient elastography. The technology exploited for ARFI has been incorporated into a conventional ultrasound system, allowing ultrasound analysis of liver morphology at the same time. In addition, ARFI allows elastography to be performed with a flexible metering box at variable depths, enabling the exact localization of the measurement site. This method can also be performed in patients with ascites meanwhile ascites and obesity are limiting factors for transient elastography.
Ascites is a common complication in patients with cirrhosis, occurring in about $60 \%$ of patients with cirrhosis over 10 years and considered a natural progression of cirrhosis $[2,3]$. According to a recent study, cirrhosis ascites accounted for $85 \%$ of the causes of ascites, other causes including malignant disease of $7.33 \%$, heart failure of $3.33 \%$, tuberculosis of $6.66 \%$, etc. [4]. Currently, there are no studies about the role of ARFI to predict cirrhotic ascites in Vietnam. Therefore, we aimed to investigate the value of Acoustic Radiation Force Impulse elastography for discriminating causes of cirrhotic ascites.

\section{Materials and Methods}

- Study design: Cross-sectional Analysis

- Population: 90 patients older than 18 yrs with ascites at Nguyen Trai hospital and Clinic of Pham Ngoc Thach University of Medicine in Vietnam from January 2013 to October 2018.

\section{- Inclusion criteria:}

- Patients agree to join the study

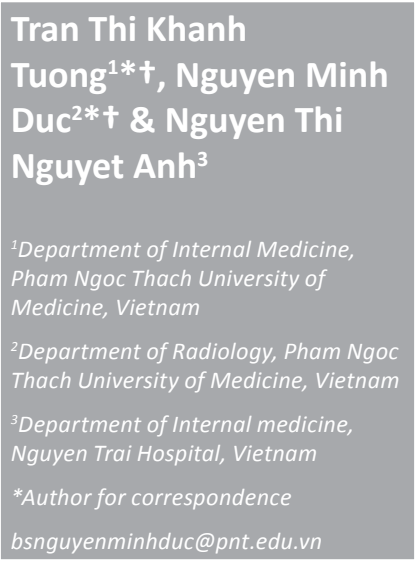


- Patients don't have contraindication of abdominal paracentesis

\section{- Exclusion criteria}

- Aminotransferase serum $>10 \times \mathrm{ULN}$

- Invalid results (ARFI): successful rate $<60 \%, \mathrm{IQR} / \mathrm{med}>30 \%$ [5].

- Criteria to diagnosis cirrhotic ascites:

- $\quad$ APRI $\geq 2$

- Ascitic fluid total protein and the serumascites albumin gradient (SAAG)>1.1 $\mathrm{g} / \mathrm{dl}$ and the ascitic total protein concentration $<2.5 \mathrm{~g} / \mathrm{dl}[2,3,6,7]$

APRI $=[($ AST $/$ ULN AST $) \times 100] /$ Platelets $\left.\left(10^{9} / \mathrm{L}\right)\right]$

- Criteria to diagnosis ascites that due to other causes:

- Tuberculous peritonitis: Adenosine deaminase (ADA) in ascitic fluid $\geq 39$ IU/L [8]

- Cancer ascites: The ascites fluid has elevated protein concentrations and a low SAAG with positive cytology or the conventional methods demonstrate the malignant tumor such as abdominal CT, laparoscopy in case of negative cytology [9].

- Ascites due to heart failure: Ascitic fluid NT-proBNP levels > 1000 pg/mL [10]

\section{- Performance:}

All patients signed an informed consent form before enrolling this study. Medical history, physical examination results, and lab tests were collected. All patients underwent ARFI elastography incorporated in Siemens ACUSON S2000 (Germany) and abdominal paracentesis. 10 ARFI measurements of the right liver through the intercostal space were performed and the median values were calculated (FIGURE 1). SWV were expressed in $\mathrm{m} / \mathrm{s}$ as median $(\mathrm{M})$, mean and standard deviation, and interquartile range (IQR), with the IQR/M ratio used to evaluate the variability of the measurements. All patients will be adopted paracentesis. It is noted that paracentesis will not be indicated in cases of acute abdomen diseases, severe thrombocytopenia (platelet count $<20 \times 103 / \mu \mathrm{L}$ ), coagulopathy (INR >2.0), pregnancy, distended urinary

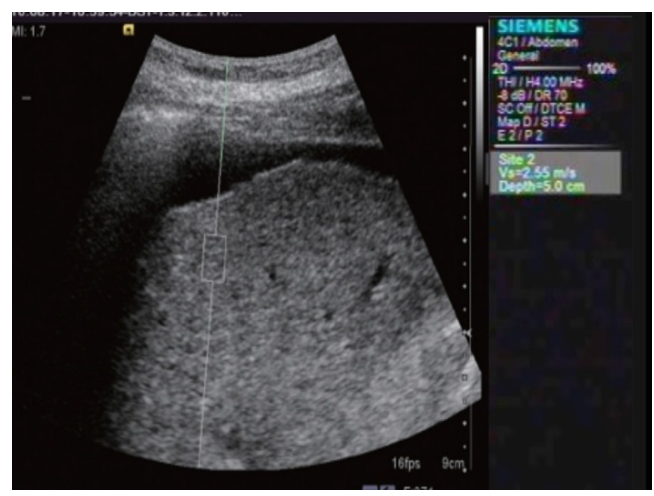

Figure 1. Liver stiffness with SWV 2.55 m/s (F4) in a patient with cirrhotic ascites.

bladder, abdominal wall cellulitis, distended bowel, intra-abdominal adhesions.

- Data management [statistic software STATA 12 (USA)] :

- To determine accuracy in diagnosis of fibrosis degree by ROC (Receiver Operating Characteristic) analysis including Area Under de ROC Curve (AUROC), Confidence Interval (CI)

- To determine optimal the cut-off value and to calculate sensitivity, specificity, PPV and NPV. The cut-off value was optimal value corresponding to the maximum Youden index (Youden index $=($ sensitivity + specificity -1$))$.

- Result is statistically significant when $\mathrm{p}$ $<0.05$.

\section{Results}

AUROC of ARFI elastography to diagnose cirrhotic ascites was 92\% (FIGURE 2).

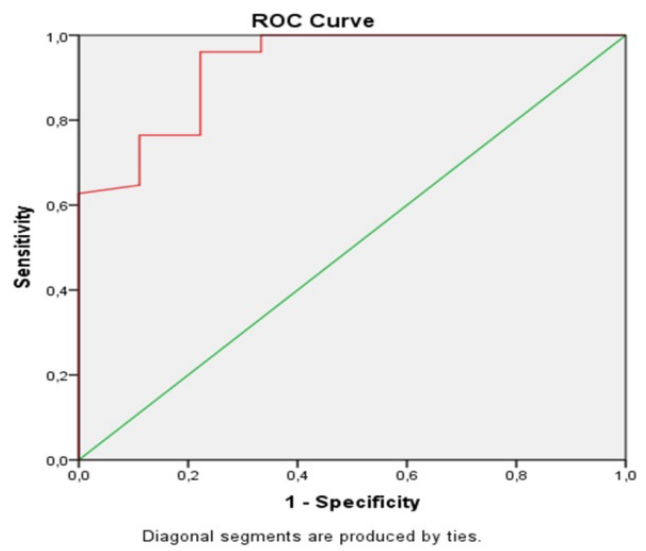

Figure 2. ROC of ARFI in diagnosis of cirrhotic ascites. 
Optimal cut-off value of SWV was $2.2 \mathrm{~m} / \mathrm{s}$ for predicting cirrhosis (and ascites in the context of cirrhosis). With this cut-off value, ARFI had sensitivity of $98 \%$, specificity of $66.7 \%$, positive predictive value of $94.3 \%$, negative predictive value of $85.71 \%$ for predicting cirrhotic ascites (TABLES 1-3).

\section{Discussion}

Adverse events related to paracentesis were leakage of ascitic fluid, infection, bleeding, and bowel perforation. Mortality is rare but has been documented. Nonetheless, in this study, the complications were not observed. There are numerous causes of ascites, but the most common cause of ascites is cirrhosis, which accounts for approximately $85 \%$ of cases [4]. Approximately $5 \%$ of patients with ascites have more than one cause, such as cirrhosis along with tuberculosis peritonitis, peritoneal carcinomatosis, heart failure, or diabetic nephropathy [4]. In our study, there were no cases that had more than one cause of ascites. $84.4 \%$ of patients had cirrhotic ascites and $15.6 \%$ of them had non-cirrhotic ascites caused by tuberculosis $(8.9 \%)$, cancer $(4.5 \%)$ and cardiac failure $(2.2 \%)$. Our findings were in line with previous studies $[4,11]$.
In practice, etiologies of ascites can be obvious from the history, physical examination and routine investigations such as blood test and abdominal ultrasound. Ultrasonography is probably the most effective modality to be able to reveal some causes of ascites. Ultrasonography may also be served as a screening test for hepatocellular carcinoma and portal hypertension, splenomegaly and portal vein thrombosis. Nonetheless, the differentiation between benign and malignant ascites by means of ultrasound is frequently difficult or impossible.

ARFI is a novel non-invasive ultrasound elastography able to assess liver stiffness in patients with ascites, therefore this new technique can be performed in addition to conventional methods. We obtained valid ARFI measurements in $90(100 \%)$ of the cases, ARFI elastography is feasible in all patients with ascites. In our study, we found statistically significant differences between SWV in patients with cirrhotic ascites versus those with noncirrhotic ascites $(3.84 \pm 1.43 \mathrm{~m} / \mathrm{s} v$ v. $1.48 \pm 0.55$ $\mathrm{m} / \mathrm{s})(P<0.001)$. Our results were confident enough to discriminate between the cirrhotic or non-cirrhotic etiology of ascites. These results

\section{Table 1. Characteristics of the patients.}

\begin{tabular}{|lccc|}
\hline Parameter & All patients & Cirrhotic group & Non-cirrhotic group \\
\hline Mean age (yr) & $54.9 \pm 12.9$ & $56.5 \pm 16.2$ & $49.6 \pm 15.9$ \\
\hline Male & $65.6 \%$ & $68.4 \%$ & $42.9 \%$ \\
\hline Mean AST(U/l) & $74.4 .5 \pm 45.3$ & $82.9 \pm 53.6$ & $33.3 \pm 24.2$ \\
\hline Mean ALT(U/l) & $47.3 \pm 35.4$ & $48.5 \pm 37.1$ & $42.6 \pm 33.1$ \\
\hline Mean APRI & $2.33 \pm 1.45$ & $2.71 \pm 1.89$ & $0.32 \pm 0.18$ \\
\hline Mean bilirubin (mg/dl) & $2.42 \pm 2.08$ & $3.56 \pm 2.92$ & $1.02 \pm 1.04$ \\
\hline Mean INR & $1.77 \pm 1.76$ & $2.03 \pm 1.54$ & $0.41 \pm 0.38$ \\
\hline Mean albumin (g/dl) & $2.92 \pm 0.67$ & $2.73 \pm 0.62$ & $4.05 \pm 0.76$ \\
\hline
\end{tabular}

\begin{tabular}{|lcc|}
\hline Table 2. Causes of ascites. & n & \% \\
\hline Cirrhosis & 76 & 84.4 \\
\hline Non-cirrhosis & & \\
- Tuberculosis & 8 & 8.9 \\
- Cancer & 4 & 4.5 \\
- Cardiac failure & 2 & 2.2 \\
\hline Total & 90 & 100 \\
\hline
\end{tabular}

\begin{tabular}{|lcccc|}
\hline Table 3. SWV of cirrhotic and non-cirrhotic ascites. & & & \\
& $\mathbf{n}$ & Mean $(\mathbf{m} / \mathbf{s})$ & SD $(\mathbf{m} / \mathbf{s})$ & $\mathbf{P}$ \\
\hline Cirrhosis & 76 & 3.84 & 1.43 & $<0.001$ \\
\hline Non-cirrhosis & 14 & 1.48 & 0.55 & $<0.001$ \\
\hline
\end{tabular}


were consistent with a study by Bota et al., [12]. In our study, AUROC had a validity of $92.1 \%$ with $95 \% \mathrm{CI}=0.963-0.982$ to diagnose of cirrhotic ascites. Cut-off value of SWV proposed by meta-analysis study for predicting compensated liver cirrhosis was $1.87 \mathrm{~m} / \mathrm{s}$ lower than our result $(2.2 \mathrm{~m} / \mathrm{s})$. This difference is owing to all cirrhotic patients without ascites presented in meta-analysis study [13].

With the optimal cut-off value of SWV of $2.2 \mathrm{~m} / \mathrm{s}$ for predicting cirrhosis (and ascites in the context of cirrhosis), ARFI had sensitivity of $98 \%$, specificity of $66.7 \%$, positive predictive value of $94.3 \%$, negative predictive value of $85.71 \%$. In a study by Bota et al., on ascitic patients, the results revealed that with the cutoff value of SWV of $2 \mathrm{~m} / \mathrm{s}$, ARFI had sensitivity of $94.5 \%$, specificity of $86.2 \%$, positive predictive value of $96.2 \%$, negative predictive value of $80.6 \%$ [12]. Thus, ARFI elastography had excellent positive predictive value (>90\%) to confirm the presence of liver cirrhosis. Furthermore, ARFI should be considered as a screening test similar to ultrasound for cirrhotic ascites owing to very high sensitivity (>90\%). According to AASLD guidelines, a diagnostic paracentesis is recommended in all patients with new onset and analysis of the ascitic fluid can help determine whether the fluid is infected or due to portal hypertension or other causes [14]. Nonetheless, this is an invasive approach that has some relative contraindications, complications and requires hospitalization. From the results of our research, ARFI elastography was efficacious to evaluate ascites and this method should be the first investigation performed after abdominal ultrasound. ARFI elastography should be carried out prior to abdominal paracentesis to assess initially etiologies of ascites because this method can manifest liver morphology, echostructure, portal hypertension, tumor lesions, and liver stiffness at the same time.

\section{Conclusion}

ARFI elastography was feasible in all patients with ascites and it had a high performance for patients with cirrhosis. ARFI plays an effective role in discrimination between cirrhotic and non-cirrhotic cause of ascites.

\section{Disclosure statement}

Tran Thi Khanh Tuong and Nguyen Minh Duc contributed equally to this article. All authors read and approved manuscript. The authors of this manuscript declare no conflict of interest.

\section{REFERENCES}

1. D'Onofrio M, Crosara S, De-Robertis R et al. Acoustic radiation force impulse of the liver. World. J. Gastroenterol. 19: 4841-4849, (2013).

2. Moore KP, Aithal GP. Guidelines on the management of ascites in cirrhosis. Gut. 55: 12, (2006).

3. Wong F. Management of ascites in cirrhosis. J. Gastroenterol. Hepatol. 27: 11-20, (2012).

4. Shaikh MA, Khan J, Almani S et al. Frequency of causes of ascites in patients admitted at medical unit of a tertiary medical care facility. J. Ayub. Med. Coll. Abbottabad. 22: 88-92, (2010).

5. Bota S, Sporea I, Sirli R et al. Factors associated with the impossibility to obtain reliable liver stiffness measurements by means of Acoustic Radiation Force Impulse (ARFI) elastography- -analysis of a cohort of 1,031 subjects. Eur. J. Radiol. 83: 268-272, (2014).

6. Biecker E. Diagnosis and therapy of ascites in liver cirrhosis. World. J. Gastroenterol. 14: 12371248, (2011).

7. Huang LL, Xia HH, Zhu SL. Ascitic Fluid Analysis in the Differential Diagnosis of Ascites: Focus on Cirrhotic Ascites. J. Clin. Transl. Hepatol. 2: 58-64, (2014).

8. Riquelme A, Calvo M, Salech F et al. Value of adenosine deaminase (ADA) in ascitic fluid for the diagnosis of tuberculous peritonitis: a metaanalysis. J. Clin. Gastroenterol. 40: 705-710, (2006).

9. Sangisetty SL, Miner TJ. Malignant ascites: A review of prognostic factors, pathophysiology and therapeutic measures. World. J. Gastrointest. Surg. 4: 87-95 (2012).

10. Sheer TA, Joo E, Runyon BA. Usefulness of serum N-terminal-ProBNP in distinguishing ascites due to cirrhosis from ascites due to heart failure. J. Clin. Gastroenterol. 44: 23-26, (2010).

11. Runyon BA. Care of patients with ascites. $N$. Engl. J. Med. 330: 337-342, (1994).

12. Bota S, Sporea I, Sirli R et al. Value of acoustic radiation force impulse elastography for the assessment of ascites syndrome. World. J. Radiol. 3: 205-209, (2011).

13. Bota S, Sporea I, Sirli R et al. How useful are ARFI elastography cut-off values proposed by meta-analysis for predicting the significant fibrosis and compensated liver cirrhosis? Med. Ultrason. 17: 200-205, (2015).

14. European Association for the Study of the Liver. EASL Clinical Practice Guidelines for the management of patients with decompensated cirrhosis. J. Hepatol. 69: 406-460, (2018). 